the weights resected. The average rate of resection was $1.01 \mathrm{~g} / \mathrm{min}$ with an average operating time of 58 minutes. The average volume of blood transfused per patient was 1.9 units, but 25 patients required no transfusion. Postoperatively a $22 \mathrm{~F}$ three-way catheter was used and the bladder irrigated with saline until the bleeding had stopped.

\section{Results}

Of the 102 patients, two died in the postoperative period. Both were in category 3 of preoperative fitness. One was an 85-year-old diabetic with a badly ischaemic leg, the other a 92-year-old man in whom a fat embolus was diagnosed post mortem. This was believed to have occurred after the prostatic capsule was perforated.

Eleven patients had urinary infections around the time of the operation and nine had positive urine cultures towards the end of the hospital stay. All infections were treated with appropriate chemotherapy. Throughout the period under review the policy was to remove the catheter on the fourth postoperative morning. In 17 patients the catheter was removed at some other time, the average being 5.6 days postoperatively. Although the average time spent in hospital after operation was 8.3 days, 21 patients were discharged on the sixth day. All patients were seen in the outpatient department one and four months after their operation. They were then discharged if symptom free but kept under review if any problems were apparent.

Ninety patients reported that they had good control of their urine with no dribbling at any time and were thus classified as having good continence. Nine patients, whose basic control was good but who suffered occasional dribbling, enuresis, or similar symptoms, were classified as having fair continence. Three patients were frankly incontinent: one was acceptably dry at 18 months, but the other two continued to need incontinence padding or an appliance.

It was our routine practice not to pass urethral sounds at the follow-up visits unless the patient complained that his stream was poor. Four patients, however, had developed urethral strictures and required between two and six dilatations. All patients had a midstream specimen of urine taker at four months and six had an infection, the offending organisms being mainly coliforms or proteus.

\section{Discussion}

Once a man with benign prostatic hypertrophy has developed appreciable bladder outflow obstruction giving episodes of acute retention or retention with overflow incontinence surgical intervention is necessary. The choice is permanent catheterisation or an operation. An operation is preferable provided it can be done safely because it offers a permanent cure in most cases, whereas an indwelling catheter represents a permanent source of inconvenience and potential danger.

The advantage of transurethral resection is that virtually nobody is too frail to withstand it, particularly with the increasing use of regional anaesthesia. We have found that the state of many patients on preoperative anaesthetic assessment is such that they would be fit for a transurethral resection but not for an open operation. Those who undergo resection can leave hospital more quickly. ${ }^{2} \mathrm{~A}$ patient whose bladder outflow obstruction is due to a large prostate need not be excluded from the benefits of transurethral resection. He may be operated on within a perfectly acceptable margin of safety and will not be exposed to any greater risk of developing a stricture or becoming incontinent. We believe that the results in this group of men, many of whom were elderly and infirm, bear this out.

The first 17 patients in the series were under the care of either Mr G C Tresidder or Professor J P Blandy at The London Hospital, and we are grateful for their permission to report these cases.

\section{References \\ ${ }^{1}$ Singh, M, Tresidder, G C, and Blandy, J P, British fournal of Urology, 1973, 45, 93. \\ ${ }^{2}$ Argyrou, S, et al, British Medical fournal, 1974, 3, 511.}

\title{
Prevalence of facial acne vulgaris in late adolescence and in adults
}

\author{
W J CUNLIFFE, D J GOULD
}

British Medical fournal, 1979, 1, 1109-1110

\section{Summary and conclusions}

A survey of 1066 healthy women and 1089 healthy men aged 18-70 years, performed to determine the prevalence of facial acne, showed that clinical acne was not confined to adolescents. Though it was more prevalent among men than women at 18 , beyond the age of 23 clinical acne was more prevalent among women as the prevalence in men gradually declined. At $40-49$ years $3 \%$ of men and $5 \%$ of women still had definite, albeit mild, clinical acne, and at $50-59$ years $6 \%$ of men and $8 \%$ of women had physiological acne.

Department of Dermatology, General Infirmary, Leeds W J CUNLIFFE, MD, FRCP, consultant dermatologist

D J GOULD, BSC, MRCP, registrar (now lecturer in dermatology, Hallamshire Hospital, Sheffield S10 2JF)
The surprisingly high prevalence of acne in adults may be related to antibiotic treatment or, in women, to the use of oral contraceptives or cosmetics, though this survey did not study their influence. Further studies in different populations are needed to establish the prevalence of acne in the community, and its distribution.

\section{Introduction}

Over the last few years we have seen an increasing number of patients aged 18 years and over with acne. This might reflect either an increasing awareness of this department's interest in acne or a genuine increase in the prevalence and severity of the disorder. An increase in prevalence might result from treatment with long-term antibiotics or, in women, from the use of cosmetics, ${ }^{1}$ or certain types of oral contraceptives.

Information on the prevalence of acne in the pre-antibiotic era is limited, ${ }^{2}$ so we cannot assess the influence of antibiotics. Pathogenic factors in patients with acne aged 18 years and above, such as the sebum excretion rate, ${ }^{3}{ }^{4}$ surface bacteria, ${ }^{5}$ and surface lipid composition, ${ }^{6}$ have also been discussed, but there is no precise information about the prevalence of acne or the age at 
which it disappears. For these reasons we have investigated the prevalence of facial acne in the population aged 18-65 years in the Leeds area.

\section{Subjects and methods}

A total of 2155 Caucasian volunteers aged 18-70 years (1066 women, $1089 \mathrm{men}$ ) from the university and hospital services, local authorities, and large industrial firms in Leeds were studied. Once patients who were receiving treatment (about $1 \%$ of the survey population) had been excluded 2133 patients were left for study. Only the severity of facial acne was assessed. All observations were conducted under excellent lighting conditions in October to April to exclude the modifying effect of ultraviolet light. We could not control the sample of women for the effects of oral contraception or variation in acne with the menstrual cycle.

The severity of acne was recorded using a subjective ranking scale from $0-10$. This scheme accounts for inflamed and non-inflamed lesions without counting their number, and concurrence of scoring between trained observers is high $(70 \%)$ with $28 \%$ of observations falling within one grade of each other.?

The information thus obtained was then regrouped into four major categories: no acne (grade 0); physiological acne (grade 1)-a few non-inflamed and inflamed lesions (usually less than five) apparent to the physician but rarely to the patient; non-inflamed and inflamed lesions (grade 2)-these lesions (usually numbering 5-10) were apparent to the patient but not sufficient to warrant clinical referral (this category was probably similar to Kligman's acne minor ${ }^{8}$ ); clinical acne requiring treatment (grades 3-10)-these grades correspond to acne major described by Kligman ${ }^{8}$ and range from superficial inflamed lesions to nodular and cystic lesions. The significance of the results was determined by the $\chi^{2}$ test.

\section{Results}

The table shows the age distribution of the volunteers, and the figure shows the prevalence of each type of acne in each age group: 18-year-olds had the highest prevalence of clinical acne on the face$35 \%$ in men and $23 \%$ in women. The prevalence then declined in

Age distribution of study population

\begin{tabular}{lcccccccccccc}
\hline Age (years) : & 18 & 19 & 20 & 21 & 22 & 23 & $24-29$ & -34 & -39 & -49 & -59 & -70 \\
\hline No of women: & 100 & 133 & 93 & 90 & 88 & 73 & 162 & 67 & 69 & 123 & 70 & 20 \\
No of men: & 66 & 107 & 57 & 60 & 58 & 44 & 198 & 123 & 83 & 127 & 95 & 25 \\
\hline
\end{tabular}

both groups, but less so among women. From 29 to 39 years women showed a significantly higher prevalence $(P<0 \cdot 05)$. Severe acne was most common in men aged $18-23$ years $(2.5 \%)$. Only $1 \%$ of women in this age group were similarly affected. The pattern was similar for grade 2 or acne minor; again the prevalence was highest at 18 years. Between 23 and 29 years women had a higher prevalence $(P<0.05)$. Acne minor was still present in patients of both sexes over 40 years. Physiological acne was most common among 30-34-year-olds in both sexes and was only significantly more common in women at 18 years $(P<0 \cdot 01)$. Acne of any type had virtually disappeared from the sample population by the age of 60 years.

Scarring was seen in $0.8 \%$ of men and $0.5 \%$ of women. The scars were of the ice-pick atrophic depressed type. In only three of the subjects was the scarring severely disfiguring.

\section{Discussion}

Knowing the age and sex distribution of a disease in the community may help to determine prognosis and enable the results of laboratory investigations to be related to the disease process. Long-term changes in the pattern of disease may also be observed and comparisons with other racial groups made.

Our investigation has confirmed the findings of Burton et al, ${ }^{9}$ that men have more severe acne than women at 18 years. This finding correlates well with the greater sebum excretion rate in men at this age. Facial acne improves in the late teens but,

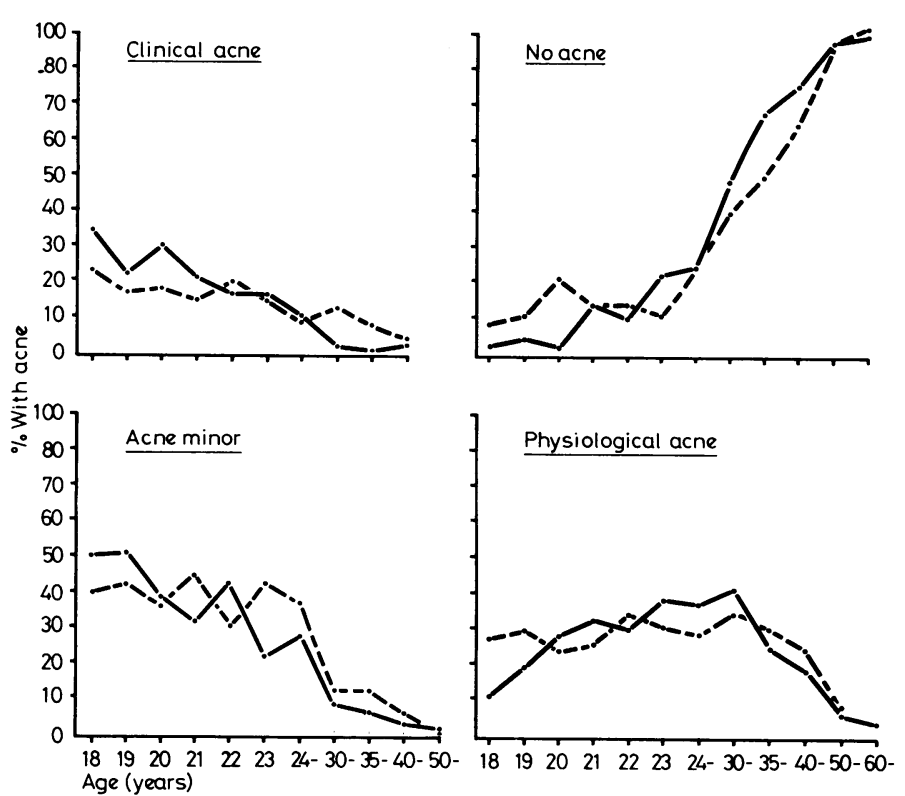

Incidence at various ages of grades of acne.

surprisingly, is still a significant clinical problem in men up to 29 years and in women up to 40 years. The greater prevalence in adult women may be related to the use of low-dose oral contraceptives, which are often androgen dominant, ${ }^{10}$ or to cosmetics, which have been shown to be acnegenic. ${ }^{1}$ The greater prevalence among women is not related to sebum excretion rate as men produce more sebum, although the sebum excretion rate has not been measured in women taking low-dose oral contraceptives. Other external factors that may affect the prevalence of acne include diet and the widespread use of antibiotics for the treatment of both acne and other diseases. The only factor we were able to exclude in our study was treatment for acne.

We hope that our investigation will encourage further studies of the prevalence of acne in different populations. Such studies may further the understanding of the genetic and environmental factors implicated in the pathogenesis of acne.

We thank Dr R Alpin, Dr R Ellis, and Mr K Senior for allowing access to patients in the various industrial concerns.

\section{References}

${ }^{1}$ Kligman, A M, and Mills, O H, jun, Archives of Dermatology, 1972, 106, 843.

${ }^{2}$ Cohen, E L, Lancet, 1942, 1, 168.

${ }^{3}$ Strauss, J S, and Pochi, P E, Advances in Biology of Skin, 1963, 4, 220

4 Cunliffe, W J, and Shuster, S, British fournal of Dermatology, 1969, 81, 697.

${ }^{5}$ Marples, R R, Downing, D T, and Kligman, A M, fournal of Investigative Dermatology, 1971, 56, 127.

${ }^{6}$ Cotterill, J A, Cunliffe, W J, and Williamson, B, British Fournal of Dermatology, 1971, 85, 130.

7 Gould, D J, and Cunliffe, W J. Personal communication.

${ }^{8}$ Kligman, A M, Fournal of Investigative Dermatology, 1974, 62, 268.

${ }^{9}$ Burton, J L, et al, British fournal of Dermatology, 1971, 85, 119.

10 Barranco, V P, Cutis, 1974, 14, 384.

ONE HUNDRED YEARS AGO At the recent meeting of the Society of German Surgeons in Beriin, Dr von Langenbeck showed a calculus weighing 600 grammes (more than 21 ounces avoirdupois) which he had removed from the body of a man who had had stricture of the urethra. The stone nearly filled the bladder; it was composed of phosphates, and no foreign body could be found as a nucleus. Dr Thiersch of Leipzic said he had successfully removed a calculus twothirds as large as that described by Dr von Langenbeck, from a man who had broken off about an inch of a paraffin bougie in his bladder. No paraffin, however, could be found in the calculus. (British Medical fournal, 1879.) 